\title{
HOMOTHALLISM EXPRESSION IN KLUYVEROMYCES LACTIS
}

\author{
C. NOVOTNY், J.-L. FUENTES, P. CARRE, H. BOZE, G. MOULIN, AND P. GALZY \\ Chaire de Génétique et Microbiologie, ENSA, 34060 Montpellier Cedex, France
}

Received 2.iii.83

SUMMARY

\begin{abstract}
Kinetics of the mating-type interconversion was observed after heterothallic nutritionally-marked stocks of Kluyveromyces lactis were mated and sporulated. Conversion to homothallism is not finished within the period of 10 days of growth on the sporulation agar. In general, the expression of homothallism in the $\alpha$ mating-type is often delayed when compared to the $a$ mating-type. Segregation patterns of tetrads suggest the functioning of a mating-type interconversion system including a mating locus and two silent storage copies of the mating-type information.
\end{abstract}

\section{INTRODUCTION}

Homothallism of yeast has lately attracted much attention. It endows monoclonal cultures of yeast with a striking power to diploidise and subsequently to sporulate. Some scientists believe that it also serves as an example of a simple differentiating system from which conclusions may be drawn about the general mechanisms controlling differentiation in multicellular eukaryotic organisms.

The mating-type locus MAT of Saccharomyces cerevisiae exists in two states, $a$ or $\alpha$, which control the ability of yeast cells to mate and sporulate (Mortimer and Hawthorne, 1969). Two genes HML $\alpha$ and $H M R a$ were found to be necessary for the conversion of mating-types, and shown to map on chromosome III, loosely linked with the MAT locus (Harashima and Oshima, 1976). Observations that both a HO hmra hmla and $\alpha H O$ hmra hmla genotypes containing only "inactive" alleles of $H M L \alpha$ and $H M R a$ had been able to switch mating-types, lead to a conclusion that the "inactive" alleles hmra and $h m l \alpha$ were equivalent to $H M L \alpha$ and $H M R a$, respectively (Naumov and Tolstrukov, 1973; Harashima et al., 1974; Harashima and Oshima, 1976). The $H O$ gene, promoting the change at the mating-type locus, maps to the left arm of chromosome IV (Klar et al., $1981 a$ ). Genetic and biochemical studies have established that the matingtype interconversion occurs by transposition of replicas of the silent matingtype information situated at the $H M L$ and $H M R$ loci, into the expressed MAT locus (Oshima and Takano, 1971; Hicks et al., 1977).

Since the cassette model of mating-type interconversion was formulated, many pieces of genetic evidence as well as convincing proof at the molecular level, have been gathered confirming this hypothesis (Klar et al., 1981a, $b$; Nasmyth et al., 1981). Its general principles also seem applicable to the mating-type interconversion in fission yeast Schizosaccharomyces pombe (Egel and Gutz, 1981).

Our knowledge of homothallism in Kluyveromyces lactis is greatly inferior. Herman and Roman (1966) identified two independent genes for

* Present address: Institute of Microbiology, Czechoslovak Academy of Sciences, Videňská, 14220, Praha, Czechoslovakia. 
homothallism, $H_{a}$ and $H_{\alpha}$, which exerted their effect by changing the respective mating-type in a certain percentage of cells. These two genes are believed to be allele-specific, one of them changing the mating-type from $a$ to $\alpha$; and the other from $\alpha$ to $a$. Such a system can be referred to as a 2-allele one, involving two active alleles $H_{a}, H_{\alpha}$ and two inactive alleles, $h_{a}, h_{\alpha}$, in contrast with the 4 -allele cassette system of $S$. cerevisiae mentioned above.

The kinetics of homothallism expression were studied in this work. Stressing common features of the 2-allele model (Herman and Roman, 1966) and of the cassette model of $S$. cerevisiae, our observations of homothallic development of tetrads of $K$. lactis bring support for the functioning of similar mating-type interconversion systems in both yeasts.

\section{MAterials AND MEthods}

Parental strains NRRL Y-1140 ( $a$ mating-type) and NRRL Y-1118 ( $\alpha$ mating-type) were obtained from Centraalbureau voor Schimmelcultures (Baarn, Holland) as CBS 2359 and CBS 6315, respectively. They should be identical with parental stocks Y-14 and Y-123, respectively, used by Herman and Roman (1966) that originated from L. J. Wickerham. Auxotrophic mutants of the original $\mathrm{Y}-1140$ unable to utilise galactose were employed in our crosses as the $a$ mating-type strains.

The nutritionally marked stocks NRRL Y-11631 (a lysine-requiring mutant of Y-1118) and NRRL Y-11630 (a histidine-requiring mutant of Y-1140) were kindly supplied by A. I. Herman (North Reg. Res. Ctr., Peoria, Ill.).

Stocks were maintained, mated and sporulated according to Herman and Roman (1966). Homothallic and heterothallic cultures were differentiated on the basis of difference in their mating response when grown on malt extract agar. Homothallic clones formed zygotes in frequencies of at least 0.005 per cent cells when grown alone, whereas heterothallic strains exhibited no mating response under these conditions but were capable of mating with $a$ - or $\alpha$-tester strains. Frequency of mating was established by searching for zygotes in the microscope.

Mating-types of spores were determined by their mating with $a$ - and $\alpha$-tester strains (original $\mathrm{Y}-1140$ and $\mathrm{Y}-1118$ ). Mating response was checked after 48 hours' growth following the cross by replica plating of the 48 hours' streaks. $a$ and $\alpha$ mating-types were segregating always in the ratio $2 / 2$ though in some tetrads this segregation was obscured by strong homothallism.

\section{Results}

\section{(i) Homothallism/heterothallism segregation}

According to Herman and Roman's hypothesis, our heterothallic parental strains had genotypes $a \mathrm{~h}_{a} H_{\alpha}$ ( $a$ mating-type; NRRL Y-1140, NRRL Y$11630)$ and $\alpha H_{a} h_{\alpha}$ ( $\alpha$ mating-type; NRRL Y-1118, NRRL Y-11631). Table 1 shows results of tetrad analyses in the crosses of $a h_{a} H_{\alpha} \times \alpha H_{a} h_{\alpha}$ strains. Frequencies of tetrad types originating in our Y-1140 $\times$ Y -1118 crosses performed within the span of as long as three years, document that the segregation pattern for homothallism is rather stable. 


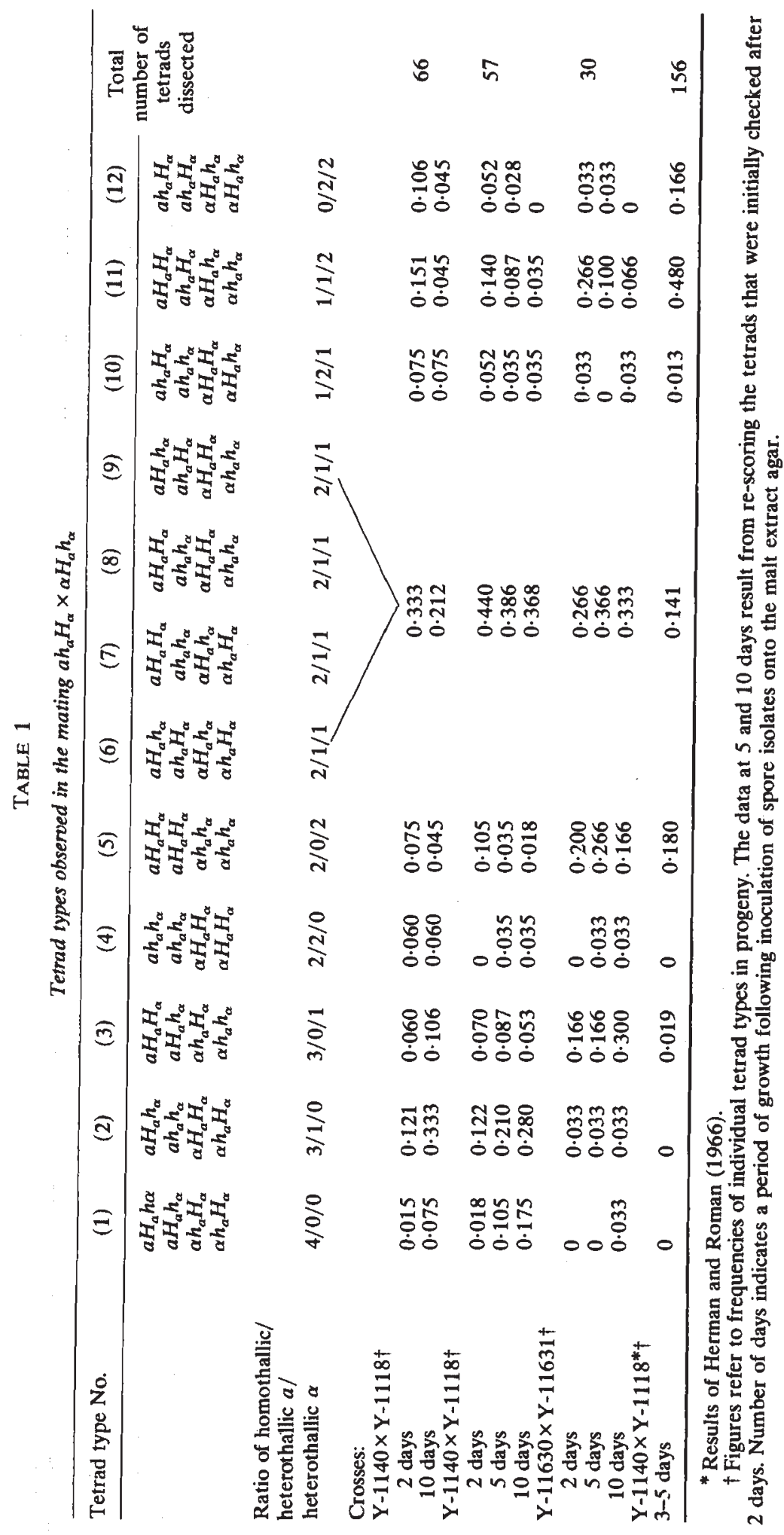




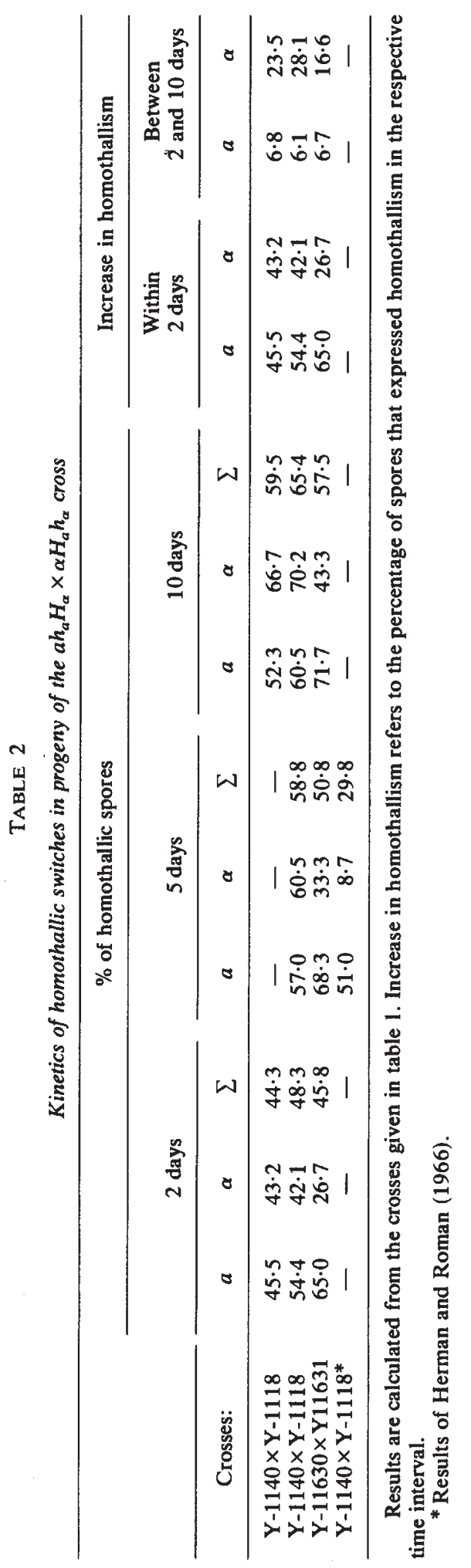




\section{(ii) Kinetics of homothallism expression}

We were surprised at the fact that some spores expressed homothallism very early after they had been inoculated on malt extract agar whereas others did so only much later. The analysis of the "rapid" homothallism (within 2 days' growth) and of the "slow" homothallism (2 to 10 days" growth) revealed inequality in the mating-type switches (table 2). The "rapid" phase is often characterised by a more frequent switch of $a$ type to homothallism, contrary to the "slow" phase, where expression of homothallism in $\alpha$ spores outnumbers the $a \rightarrow \alpha$ switch 2.5 to 4.6 times. Rates of $\alpha$ switches in related stocks vary more significantly than those of $a$ switches. Reasons for the difference between the $a$ and $\alpha$ switches are unknown.

Comparing individual rates of $a$ and $\alpha$ switches to homothallism in the crosses $\mathrm{Y}-1140 \times \mathrm{Y}-1118$ and $\mathrm{Y}-11630 \times \mathrm{Y}-11631$, they differ to a greater extent than the respective overall rates. The overall rate of homothallism found by Herman and Roman (1966) was, however, much lower due to a very low rate of $\alpha$ switch to homothallism in the progeny of their cross (table 2).

\section{(iii) Linkage of $\alpha$ and $h_{\alpha}$ genes}

Herman and Roman (1966) found strong linkage between genes $\alpha$ and $h_{\alpha}$ (9 per cent recombination). Assuming the function of $H_{\alpha}$ and $H_{a}$ genes according to the 2-allele concept, our crosses of table 1 show a very loose linkage between the genes $\alpha$ and $h_{\alpha}$ (30 to 40 per cent recombination) when scored at 2 days. The data at 10 days, i.e., after the "slow" phase of homothallism expression, effectively shows no linkage between $\alpha$ and $h_{\alpha}$ ( $>42$ per cent recombination).

\section{(iv) Cassette-model behaviour of mating-type interconversions}

The 4-allele cassette model of $S$. cerevisiae identifies the inactive alleles $h_{a}$ and $h_{\alpha}$ with $H_{\alpha}$ and $H_{a}$ active ones respectively (table 3 ). Such an assumption would change the expected segregation pattern of our crosses, making some phenotypic groups missing (table 4). Thus the tetrads with segregation ratios of homothallic/heterothallic $a /$ heterothallic $\alpha$ spores $3 / 1 / 0,3 / 0 / 1,2 / 2 / 0,2 / 0 / 2,2 / 1 / 1,1 / 2 / 1$, and $1 / 1 / 2$ must be considered as $4 / 0 / 0,4 / 0 / 0,4 / 0 / 0,4 / 0 / 0,4 / 0 / 0$ or $3 / 0 / 1$ or $3 / 1 / 0,2 / 1 / 1$, and $2 / 1 / 1$ phenotypes, respectively. Provided that all the progeny that are able to

TABLE 3

The activity of $H_{a} / h_{a}$ and $H_{\alpha} / h_{\alpha}$ alleles in the 2-and 4-allele model

\begin{tabular}{|c|c|c|}
\hline & \multicolumn{2}{|c|}{ Allele activity } \\
\hline Allele & 2-allele model & 4-allele model \\
\hline $\begin{array}{l}H_{a} \\
h_{a} \\
H_{\alpha} \\
h_{\alpha}\end{array}$ & $\begin{array}{c}a \rightarrow \alpha \text { switch } \\
\text { no switch } \\
\alpha \rightarrow a \text { switch } \\
\text { no switch }\end{array}$ & $\begin{array}{l}a \rightarrow \alpha \text { switch; silent } \alpha \text { information } \\
\alpha \rightarrow a \text { switch; silent } a \text { information } \\
\alpha \rightarrow a \text { switch; silent } a \text { information } \\
a \rightarrow \alpha \text { switch; silent } \alpha \text { information }\end{array}$ \\
\hline
\end{tabular}




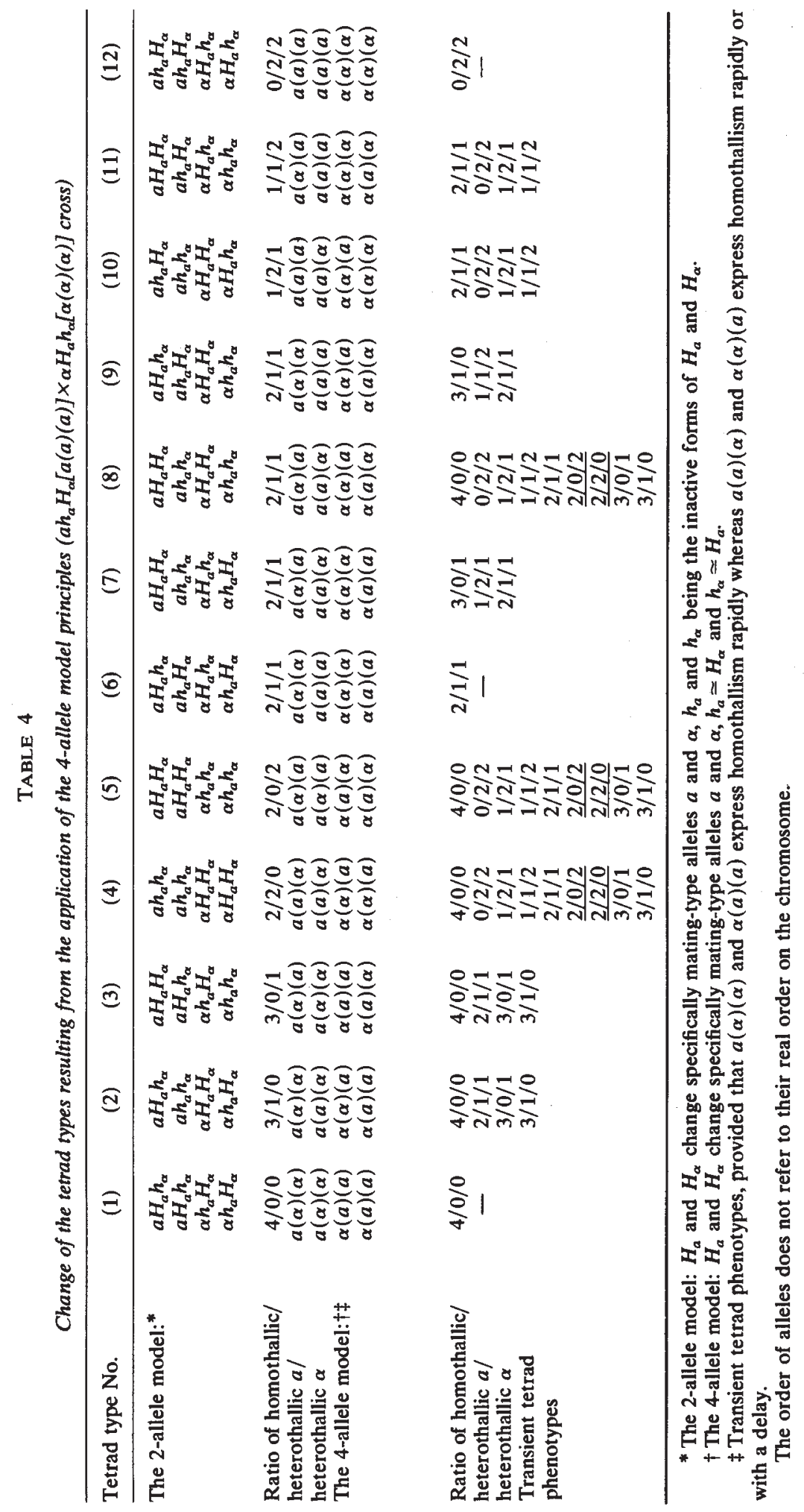


would express their homothallism, the tetrads $2 / 2 / 0,2 / 0 / 2,1 / 2 / 1$, and $1 / 1 / 2$ should not exist.

In fact, these "forbidden" tetrads were quite numerous even after 10 days' growth on malt extract agar (table 1). Our observations suggesting that some spore isolates exhibit the homothallic switch beyond 10 days of growth made us, however, re-test these tetrads for homothallism. The spore isolates had been stored for 3 months at $4^{\circ} \mathrm{C}$ before this new test was performed. Results in table 5 document that all the tetrads incompatible with the 4-allele model shifted towards higher values of homothallic/heterothallic ratio, turning these tetrads into other tetrad phenotypes which were compatible with the 4-allele model. Eventual absence of these "forbidden" phenotypes in progeny (table 4) is consistent with the 4-allelemodel behaviour in $K$. lactis.

TABLE 5

Development of tetrads incompatible with the cassette model

\begin{tabular}{|c|c|c|}
\hline \multirow[b]{2}{*}{ Cross } & \multicolumn{2}{|c|}{ Spore ratio in tetrads } \\
\hline & $\begin{array}{c}\text { Ratio of homothallic/ } \\
\text { heterothallic } a / \\
\text { heterothallic } \alpha \\
\text { after } 10 \text { days of growth }\end{array}$ & $\begin{array}{l}\text { Ratio of homothallic/ } \\
\text { heterothallic } a / \\
\text { heterothallic } \alpha \\
\text { after } 3 \text { months of } \\
\text { conservation of } \\
\text { spores at } 4^{\circ} \mathrm{C}\end{array}$ \\
\hline$Y-11630 \times Y-11631$ & $\begin{array}{l}4 / 0 / 0(1) \\
3 / 1 / 0(1) \\
3 / 0 / 1(8) \\
2 / 1 / 1(10)\end{array}$ & $\begin{array}{l}4 / 0 / 0(1) \\
3 / 1 / 0(1) \\
4 / 0 / 0(6) \\
3 / 0 / 1(2) \\
4 / 0 / 0(6) \\
3 / 0 / 1(3) \\
2 / 1 / 1(1)\end{array}$ \\
\hline & $\begin{array}{l}0 / 2 / 2 \quad(0) \\
2 / 2 / 0(1) \\
2 / 0 / 2 \quad(5) \\
1 / 2 / 1 \quad(1) \\
1 / 1 / 2 \quad(2)\end{array}$ & $\begin{array}{l}-\overline{0}(1) \\
4 / 0 / 0 \\
4 / 0 / 0(5) \\
4 / 0 / 0(1) \\
4 / 0 / 0(2)\end{array}$ \\
\hline
\end{tabular}

Numbers of asci are given in brackets.

\section{Discussion}

Though the molecular mechanism of mating-type switching in yeast is quite well understood, our knowledge of the regulation of this important biological phenomenon is somewhat meagre (Herskowitz et al., 1977; Klar et al., 1981a; Nasmyth et al., 1981).

In their pioneer work on homothallism of $K$. lactis, Herman and Roman (1966) defined the genetic system governing homothallism as comprising two mating-type-specific genes $H_{a}$ and $H_{\alpha}$, able to evoke the homothallic switch of the respective mating-type allele. $h_{a}$ and $h_{\alpha}$ were supposed to be the inactive forms of these genes. This 2-allele hypothesis showed fruitful even though several crosses were difficult to reconcile with its clear-cut predictions. Our results suggest that these discrepancies can be overcome by applying general principles of the 4-allele model for homothallism in $S$. cerevisiae (Herskowitz et al., 1977). 
The adaptation of the $S$. cerevisiae model to $K$. lactis extends the 2-allele scheme by attributing properties of functional but silent (i.e., non-expressed) $a$ and $\alpha$ information to the alleles $h_{a}$ and $h_{\alpha}$, respectively, making them fully capable of changing the expressed mating-type of the homothallic cell (table 3). Such a model modifies the segregation pattern of the $a h_{a} H_{\alpha} \times$ $\alpha H_{a} h_{\alpha}$ cross by postulating the absence of the tetrad types $2 / 2 / 0,2 / 0 / 2$, $1 / 2 / 1$, and $1 / 1 / 2$ in progeny (table 4 ). Our observations confirm this hypothesis, showing all the incompatible tetrads to disappear as homothallism was evolving in the course of time (table 5).

The validity of the new model for $K$. lactis could be further verified in the following crosses. It should be recalled in this connection that a 3/0/1 ascus refers to the tetrade type 7 (see table 4 ) according to the 4 -allele model in comparison with the 2 -allele model where it indicates the tetrade type 3 of the same table. Similarly, a $3 / 1 / 0$ ascus indicates the tetrad type 9 in the 4 -allele model and the tetrade type 2 in the 2 -allele model. 1 . A cross of the $\alpha$-heterothallic spore isolated from a $3 / 0 / 1$ ascus with the heterothallic parent $a h_{a} H_{\alpha}$ should be a parental cross $a h_{a} H_{\alpha} \times \alpha H_{a} h_{\alpha}$ yielding all the tetrad types issuing from the 4 -allele concept (see table 4 ). The 2-allele model would predict a cross $a h_{a} H_{\alpha} \times a h_{a} h_{\alpha}$ in this case, with progeny of the $2 / 2 / 0,1 / 2 / 1$ and $0 / 2 / 2$ type; 2 . a cross of the $a$-heterothallic spore isolated from a $3 / 1 / 0$ ascus with the heterothallic parent $\alpha H_{a} h_{\alpha}$ should also be a parental cross $a h_{a} H_{\alpha} \times \alpha H_{a} h_{\alpha}$ according to the 4-allele concept, giving all the tetrad types shown in table 4 . In this case the 2 -allele model would predict a cross $a h_{a} h_{\alpha} \times \alpha H_{a} h_{\alpha}$ with progeny of the $2 / 0 / 2,1 / 1 / 2$ and $0 / 2 / 2$ tetrad type; 3 . self-sporulated tetrads obtained from either of the two $\alpha$ homothallic spores originating from a $3 / 1 / 0$ ascus or from either of the two $a$ homothallic spores orginating from a $3 / 0 / 1$ ascus should be exclusively of the 4/0/0 type according to the 4 -allele model. The 2 -allele model postulates these self-sporulated tetrads to be either of the $4 / 0 / 0$ or of the $2 / 2 / 0$ type for the $3 / 1 / 0$ ascus and either of the $4 / 0 / 0$ or of the $2 / 0 / 2$ type for the $3 / 0 / 1$ ascus, according to the genotype of the respective homothallic spore.

Owing to the existence of delayed homothallic switches (table 5), the tetrads employed in these matings must be properly checked through at least several months to make sure of their homothallic/heterothallic $a /$ heterothallic $\alpha$ ratio. Such testing is now in progress to prepare stocks for these ultimate crosses.

Fortunately, self-sporulated tetrads obtained from two homothallic $a H_{a} h_{\alpha}$ spores originating from independent $3 / 0 / 1$ asci were already isolated by Herman and Roman (1966) (homothallic spores 155C, 338C). The $4 / 0 / 0$ phenotypes obtained were in contradiction with their theory expecting only the $2 / 0 / 2$ phenotypes in progeny. On the other hand, the 4 -allele model identifies the $a$ homothallic spores of the $3 / 0 / 1$ asci with the genotypes $a(\alpha)(a)^{*}$ and $a(a)(\alpha)^{*}$, and predicts only the $4 / 0 / 0$ progeny by this self-sporulation, which was actually found by Herman and Roman (1966). We consider these data provide further support for our concept of homothallism in $K$. lactis.

The back-cross $155 \mathrm{C} \times \mathrm{Y} 123 a(a)(\alpha) \times \alpha(\alpha)(\alpha)^{1}$ made by Herman and Roman (1966), yielding the $2 / 0 / 2$ tetrads, is consistent with both the

\footnotetext{
* The order of alleles does not refer to their real order on the chromosome.
} 
2-allele and 4-allele model, the latter predicting the $2 / 0 / 2,3 / 0 / 1$ and $4 / 0 / 0$ tetrads in progeny, theoretically. Lack of the two latter tetrad types (all 6 tetrads isolated were 2/0/2) may be due to a delay in the expression of homothallism in $\alpha$ clones (cf. table 2). In our opinion, such a delay may also explain a lesser number of homothallic $\alpha$ clones than of $a$ ones, observed with some strains. In addition, the $\alpha$ clones are also handicapped, in comparison with the $a$ ones, at the level of homothallism expression, for numbers of their homothallic zygotes are mostly inferior (Herman and Roman, 1966). One can think of a common mechanism being responsible for these two phenomena. However, lack of knowledge on the regulation of homothallic switches in $K$. lactis makes speculations on the possible nature of this mechanism difficult.

All other crosses performed by Herman and Roman (1966) are also well compatible with the proposed 4-allele model on condition that some tetrads must be considered still incompletely developed as to the switch of several $\alpha$ clones to homothallism (tetrads 157 and 1009 have phenotypes incompatible with the segregation pattern of the $\alpha(\alpha)(\alpha) \times a(a)(a)$ cross according to the 4-allele model, cf. table 4).

Data on the kinetics of homothallism expression (table 2) revealed that some $a$ and $\alpha$ clones expressed homothallism quite rapidly, whereas in others the expression was delayed. Putting aside for a moment the differences between the $a$ and $\alpha$ clones discussed above, an interesting point remains concerning the mode of development of tetrads to homothallism. Our observation that all tetrads exhibiting the phenotypes $2 / 0 / 2$ or $2 / 2 / 0$ at any moment of their development have the true phenotypes $4 / 0 / 0$ suggests that $a(a)(\alpha)^{*}$ and $\alpha(a)(\alpha)^{*}$ clones may delay the homothallism expression under some circumstances. Looking at the genotypes of tetrad groups in the 4-allele model (table 4), provided that only the $a(a)(\alpha)^{*}$ and $\alpha(a)(\alpha)^{*}$ genotypes and not the $a(\alpha)(\alpha)$ and $\alpha(a)(a)$ ones delay their homothallic response, the $2 / 0 / 2$ and $2 / 2 / 0$ transient phenotypes occur theoretically only in the homothallic development of three of the 4/0/0 tetrads. Such behaviour of the $a(a)(\alpha)$ and $\alpha(a)(\alpha)$ genotypes could be comprehensible in the light of findings in $S$. cerevisiae, where the position of the silent information whether at HML or HMR has a striking effect on the frequency of homothallic switches in the clone (Klar et al., 1982). Provided that the less efficient homothallic switch caused by the inferior position of the switching allele was also more time-consuming, we would have an example of a mechanism in operation responsible for this behaviour.

One can thus hypothesize that the $a(\alpha)(\alpha)$ and $\alpha(a)(a)$ clones would switch their mating-type rapidly, whereas the $a(a)(\alpha)$ and $\alpha(a)(\alpha)$ ones could display either rapid or delayed switching, according to the respective position of the opposite switching allele. Of course, this behaviour allows for a distinct developmental pattern of individual tetrad groups given in table 4 . When this theoretical developmental pattern was compared with that found in the cross $a(a)(a) \times \alpha(\alpha)(\alpha)(Y-11630 \times Y-11631)$, the development of all tetrads including the $2 / 2 / 0,2 / 0 / 2,1 / 2 / 1$, and $1 / 1 / 2$ ones fully obeyed the theoretical pattern. More observations, however, are necessary to check this hypothesis and explain the delayed expression of homothallism.

* The order of alleles does not refer to their real order on the chromosome. 
The 4-allele cassette model of mating-type interconversion in $S$. cerevisiae also involves the $H O$ gene in addition to the mating locus and 2 cryptic alleles at both the $H M L$ and $H M R$ loci. The $H O$ allele acts to increase the frequency of mating-type interconversion in contrast with its inactive allele ho. Cells containing the ho allele switch their mating-types at a frequency of approximately $10^{-6}$ (Hicks et al., 1977). If similar rates hold true for $K$. lactis, then all our heterothallic parents $\mathrm{Y}-1140, \mathrm{Y}-1118$, $Y-11630$, and $Y-11631$ must contain an HO-type allele because the $4 / 0$ and $3 / 1$ homothallic/heterothallic tetrads were found in both crosses (table 1).

No zygotes were ever found with heterothallic parental strains, indicating that matings between cells of the same mating-type did not influence our results.

We conclude that segregation patterns obtained in the crosses with $K$. lactis favour the functioning of a mating-type interconversion system that includes a mating locus and 2 silent storage copies of the mating-type information. The asymmetry seems to be a feature of this system that manifests itself in the slower onset of the $\alpha \rightarrow a$ homothallic switch, the lower percent homothallism in $\alpha$ cells (the ratio of homothallic zygotes/100 vegetative cells), and in a tendency to decrease the percent-homothallism value in $\alpha$ cells (but not in $a$ cells) following additional meioses.

Acknowledgements. The authors gratefully acknowledge Professor H. L. Roman for his helpful comments on the manuscript.

\section{REFERENCES}

EGEL, R. AND GUTZ, H. 1981. Gene activation by copy transposition in mating-type switching of a homothallic fission yeast. Current Genet., 3, 5-12.

HARASHIMA, S., NOGI, Y. AND OSHIMA, Y. 1974. The genetic system controlling homothallism in Saccharomyces yeast. Genetics, 77, 639-650.

HARASHIMA, S. AND OSHIMA Y. 1976. Mapping of the homothallic genes $H M \alpha$ and $H M a$ in Saccharomyces yeasts. Genetics, 84, 437-451.

HERMAN, A. AND ROMAN, H. L. 1966. Allele specific determinants of homothallism in Saccharomyces lactis. Genetics, 53, 727-740.

HERSKOWITZ, I., STRATHERN, J. N., HICKS, J. B. AND RINE, J. 1977. Mating-type interconversion in yeast and its relationship to development in higher eucaryotes. In Wilcox, G., Abelson, J., and Fox, C. F. (eds.) Eucaryotic Genetic Systems, ICN-UCLA Symp. on Molec. and Cellul. Biology, Vol. 8, Academic Press, New York, pp. 193-202.

HICKS, J. B., STRATHERN, J. N. AND HERSKOWITZ, 1. 1977. The casette model of mating -type interconversion. In Bukhari, A., Shapiro, J., and Adhya, S. (eds.) DNA Insertion Elements, Plasmids and Episomes, Cold Spring Harbor Laboratory, New York, pp. 457-462.

KLAR, A. J. S., HICKS, J. B. AND STRATHERN, J. N. 1982. Directionality of yeast mating-type interconversion. Cell, 28, 551-561.

KLAR, A. J. S., STRATHERN, J. N. AND HICKS, J. B. $1981 a$. A position-effect control for gene transposition: State of expression of yeast mating-type genes affects their ability to switch. Cell, 25 517-524.

KLAR, A. J. S., STRATHERN, J. N., BROACH, J. R. AND HICKS, J. B. $1981 b$. Regulation of transcription in expressed and unexpressed mating-type cassettes of yeast. Nature, 289 , 239-243.

MORTIMER, R. K. AND HAWTHORNE, D. C. 1969. Yeast genetics. In Rose, A. H., and Harrison, J. S. (eds.) The Yeasts, Part 1, Academic Press, New York, pp. 385-460.

NASMYTH, K. A., TATCHELl, K., HALL, B. D., ASTELL, C. AND SMITH, M. 1981. A position effect in the control of transcription at yeast mating-type loci Nature, 289, 244-249. 
NAUMOV, G. I. AND TOLSTORUKOV, I. 1. 1973. Comparative genetics of yeast. X. Reidentificatiuon of mutators of mating-types in Saccharomyces. Genetika, 9, 82-91.

OSHIMA, Y. AND TAKANO, 1. 1971. Mating-types in Saccharomyces: Their convertibility and homothallism. Genetics, 67, 327-335. 